\title{
Antigenicity and immunogenicity of a novel, acute HIV-1 Tanzanian subtype C gp145 envelope protein for clinical development
}

\author{
V Polonis ${ }^{1 *}$, L Wieczorek², V Kalyanaraman ${ }^{3}$, G Matyas ${ }^{1}$, S Whitney ${ }^{3}$, C Williams $^{4}$, S Tovanabutra ${ }^{2}$, E Sanders-Buell ${ }^{2}$, \\ M Wesberry ${ }^{2}$, C Ochsenbauer ${ }^{5}$, A Chenine ${ }^{2}$, M Rao ${ }^{1}$, T Tong $^{2}$, C Alving ${ }^{1}, \mathrm{H}_{\text {Cheng }}{ }^{6}$, S Zolla-Pazner ${ }^{4}$, N Michael', \\ T VanCott ${ }^{3}$, M Marovich $^{1}$
}

From AIDS Vaccine 2012

Boston, MA, USA. 9-12 September 2012

\section{Background}

Eliciting broadly reactive neutralizing antibodies remains a challenge in HIV-1 vaccine development, complicated by variations in envelope (Env) subtype and structure, and by the assays used for product down-selection. Since a majority of new HIV-1 infections are subtype C and considering the novel properties of $C$ Envs, a $C$ Env (CO6980v0c22) from an acutely infected Tanzanian was developed as a candidate HIV vaccine.

\section{Methods}

The CO6980v0c22 Env sequence was codon optimized and a stable $\mathrm{CHO}$ cell line expressing gp145 was established. Purified gp145 was adjuvanted in alum or lipid A-liposomes, injected into New Zealand white rabbits (4/group; 25 ug at weeks 0,4 , and 8 ), or BALB/c mice (5/group; $10 \mathrm{ug}$ in liposomes at weeks $0,3,6,8$ ). Antibody titers were assessed by ELISA and neutralizing antibodies were measured against pseudoviruses in TZM-bl cells or against infectious molecular clones (IMC) in a PBMC assay.

\section{Results}

Secreted gp145 is a novel subunit with the full MPER extended by three lysines. Unlike some gp140 subunits, the $4 \mathrm{E} 10$ neutralizing monoclonal antibody $(\mathrm{mAb})$ binds to gp145. IgG1b12 binds weakly, VRC01 binds potently, as does the V2-specific 697D mAb; the gp145 also binds to alpha4beta7 receptor, as demonstrated by flow cytometry. At week 10 post-immunization, rabbit sera showed strong binding antibody titers to several Env antigens, including the clade B V1V2gp70 scaffold protein. While neutralization of the HIV-2 MPER-scaffold pseudovirus was negative, cross-clade neutralization was observed in both rabbits and mice, against Tier 1 subtype $B$ and $C$ pseudoviruses, and against Tier 1 and Tier 2 IMC. Using EGS cross-linking, it appears that the majority of the gp 145 multimers are trimeric; this is currently under investigation using electron microscopy techniques.

\section{Conclusion}

These data indicate essential immunogenic features of a novel acute C HIV-1 Env that warrants further testing for potential clinical development.

\section{Author details}

${ }^{1}$ Walter Reed Institute of Research, DIV Retrovirology, Silver Spring, MD, USA. ${ }^{2}$ Henry M. Jackson Foundation, Silver Spring, MD, USA. ${ }^{3}$ Advanced Biosciences Laboratories, Inc, Rockville, MD, USA. ${ }^{4} \mathrm{New}$ York University School of Medicine, New York, NY, USA. 'University of Alabama, Birmingham, AL, USA. ${ }^{6}$ University of California, Davis, CA, USA.

Published: 13 September 2012

\section{doi:10.1186/1742-4690-9-S2-P323}

Cite this article as: Polonis et al:: Antigenicity and immunogenicity of a novel, acute HIV-1 Tanzanian subtype C gp145 envelope protein for clinical development. Retrovirology 2012 9(Suppl 2):P323. 